Article

\title{
Flexible Electrode by Hydrographic Printing for Surface Electromyography Monitoring
}

\author{
Xiong Zeng ${ }^{1}$, Ying Dong ${ }^{1, *}$ and Xiaohao Wang ${ }^{1,2}$ \\ 1 Tsinghua Shenzhen International Graduate School, Tsinghua University, Shenzhen 518055, China; \\ zengx17@mails.tsinghua.edu.cn (X.Z.); wang.xiaohao@sz.tsinghua.edu.cn (X.W.) \\ 2 Tsinghua-Berkeley Shenzhen Institute, Tsinghua University, Shenzhen 518055, China \\ * Correspondence: dongy@tsinghua.edu.cn; Tel.: +86-755-26032505
}

Received: 8 April 2020; Accepted: 15 May 2020; Published: 19 May 2020

\begin{abstract}
Surface electromyography (sEMG) monitoring has recently inspired new applications in the field of patient diagnose, rehabilitation therapy, man-machine-interface and prosthesis control. However, conventional wet electrodes for sEMG recording cannot fully satisfy the requirements of these applications because they are based on rigid metals and conductive gels that cause signal quality attenuation, motion artifact and skin allergy. In this study, a novel flexible dry electrode is presented for sEMG monitoring. The electrode is fabricated by screen-printing a silver-eutectic gallium-indium system over a transfer tattoo paper, which is then hydrographically printed on 3D surface or human skin. Peano curve in open-network pattern is adopted to enhance the mechanics of the electrode. Hydrographic printing enables the electrode to attach to skin intimately and conformably, meanwhile assures better mechanical and electrical properties and therefore improves the signal quality and long-term wearability of the electrode. By recording sEMG signal of biceps under three kinds of movement with comparison to conventional wet electrode, the feasibility of the presented flexible dry electrode for sEMG monitoring was proved.
\end{abstract}

Keywords: dry electrode; flexible device; fractal pattern; hydrographic printing; sEMG

\section{Introduction}

Flexible devices or epidermal electronics are newly developed wearable electronics, which are much more lightweight, robust and flexible in contrast to traditional devices, therefore allowing them intimately mounted on human skin [1-5]. These skin-like sensing systems provide opportunities for long-term and continuous measurement of bioelectrical signals such as electrocardiogram (ECG), electroophthalmic (EOG), electromyography (EMG) and physical parameters like temperature and humidity [5-9].

EMG signal is generated by the electrical activity of the muscle fibers that are activated almost synchronously by the motor neuron innervating them [10-12]. EMG can be detected by intramuscular electrodes [13], known as needle EMG or by electrodes mounted on skin [14], known as surface Electromyography (sEMG). As one of the most common signals non-invasive, sEMG is widely used to not only detect neurophysiology and analyze movement or gait, but also interface with man-machine and control prosthesis [8,15-17].

The most common and commercially available electrodes for bioelectrical signals acquisition are wet conductive gel electrodes. While they are characterized by causing irritation and allergy, signal degradation during long-term monitoring, as well as difficulty in adhering skin without adhesive. Dry flexible electrode is a promising alternative as they can attach to skin with intimate attachment, less motion artifact and no skin reaction [18]. While most dry flexible electrodes are typically based on polymer [19] (PDMS [20], PI [21]), metal (Au [22], AgNP [23]), carbon [24,25] and 
liquid metal (AlGaN/GaN [26], EGaIn [27-29]). Previous works also typically proved that rigid metal materials $(\mathrm{Au}$ [30], $\mathrm{Cu}$ [31]) with serpentine and bridge structure [32] could better tolerate mechanical deformations such as stretching, compressing and twisting. These materials are usually fabricated through lithography technology, chemical vapor deposition (CVD), physical vapor deposition (PVD) and other patterning techniques that are expensive and require complex processes and high-quality conditions [33-36]. While hydrographic printing technology transferring the pattern and material on the medium through water is uncomplicated and inexpensive, which has been used to process flexible electrodes on skin [37-41].

In this work, a novel flexible dry electrode for sEMG monitoring is presented. In the following sections, the pattern, structure, fabrication process and characterization of the electrode are introduced. Then the contact impedance and the stretch-resistance characteristics of the electrode are tested. Finally, the feasibility of the electrode for sEMG monitoring is verified through recording sEMG signal of biceps under three kinds of movement and testing the long term wearability in comparison to traditional wet electrode.

\section{Pattern and Structure}

Considering the generation and location of sEMG, the electrode pattern and size are well designed according to the guideline of SENIAM (surface electromyography for the non-invasive assessment of muscles) project. The electrode pattern consists of three phases, i.e., reference (REF), ground (GND), record (REC) phase, as shown in Figure 1a, from left to right. To assure that the electrode remains functional under strains of up to $30 \%$ which is the typical stretch ability of natural human skin and high signal noise ratio, each phase shapes in the first-order Peano curve [42], with the total dimensions of $5 \mathrm{~mm} \times 5 \mathrm{~mm}$ and wire width of $100 \mu \mathrm{m}$. The end pad of each phase, with dimension of $1 \mathrm{~mm} \times$ $2 \mathrm{~mm}$, provides an electrical interface for data acquisition through ACF connector (M-505A, JieMai precision automation co. LTD, ShenZhen, China). The electrode pattern is printed on flexible tattoo paper (silhouette temporary tattoo paper, American, INC, Lindon, UT, USA) that is composed of an adhesive sheet and a support sheet. The adhesive sheet contains slip layer, glue layer and support paper layer, as shown in Figure 1b. The glue layer is able to assure the electrodes intimately attaching to skin. While used as the loading carrier of the electrodes, the support sheet usually includes ethyl cellulose (EC) layer, soluble layer and support paper layer, as shown in Figure 1c. There will only be the EC layer support the pattern and material left after dissolution. With the employment of the paper material, the pattern and structure have the ability to intimately attach to skin surface without damage, meanwhile block the oxygen influencing the sensitive complex system.

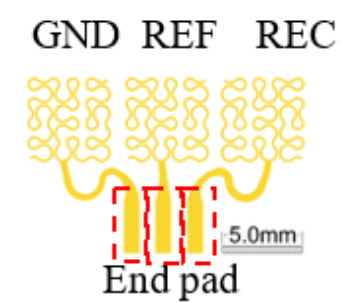

(a)

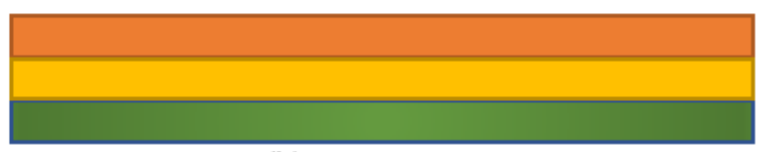

(b)

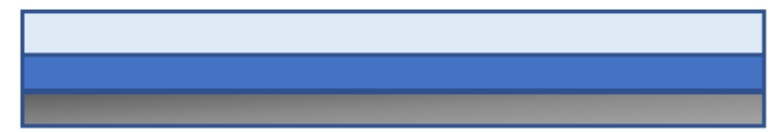

(c)

\author{
Slip layer \\ Glue layer \\ Paper layer \\ EC layer \\ Soluble layer \\ Paper layer
}

Figure 1. The fractal pattern of the flexible electrodes and the transfer tattoo paper. (a) overall pattern of the first-order Peano curve. (b) the adhesive sheet and (c) support sheet of the transfer tattoo paper.

\section{Processes and Characterization}

The electrode is manufactured by hydrographic printing silver-indium-gallium alloy system (silver, ET-5A, UVTM, $3.9 \mathrm{GPa}$; indium-gallium, $24 \%-76 \%$ ) on the tattoo paper. It has been found out that ternary alloy system silver-indium-gallium would form when silver is added into eutectic Ga-In at atmosphere condition [43]. Weak acid can be used to clean the alloy system if there are extra 
materials. As illustrated in Figure 2, the hydrographic printing process is composed of six steps. First, the adhesive sheet was laser (current $5 \mathrm{~A}$, velocity $5 \mathrm{~m} / \mathrm{s}$ ) patterned and the silver-indium-gallium alloy system was screen-printing patterned on the support sheet. After the alloy system solidified, the adhesive sheet and the support sheet were bonded by the glue layer. Then the bonding system was soaked in water and after $30 \mathrm{~s}$, the water-soluble middle layer of the tattoo paper substrate dissolved and separated the flexible film from the support paper. The left flexible film then attached to a 3D object or skin, but the surface of the flexible film was still moist. In order to reduce the oxidation of the alloy system due to the moist, it is better to take about $5 \mathrm{~min}$ to get the wet surface dry out in ambience. Moreover, the time can be shortened if heater is used.

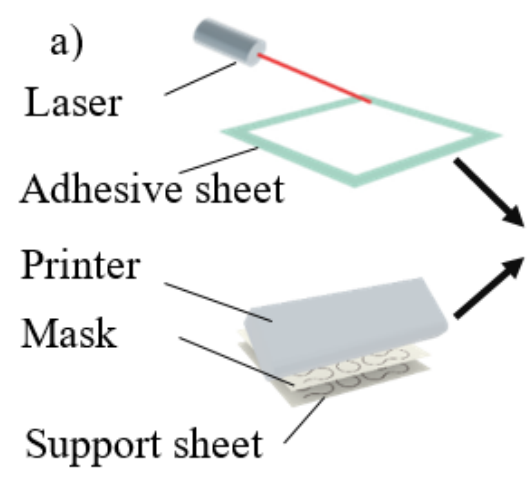

f)

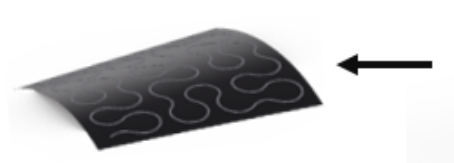

b) Slip layer + Glue layer c)

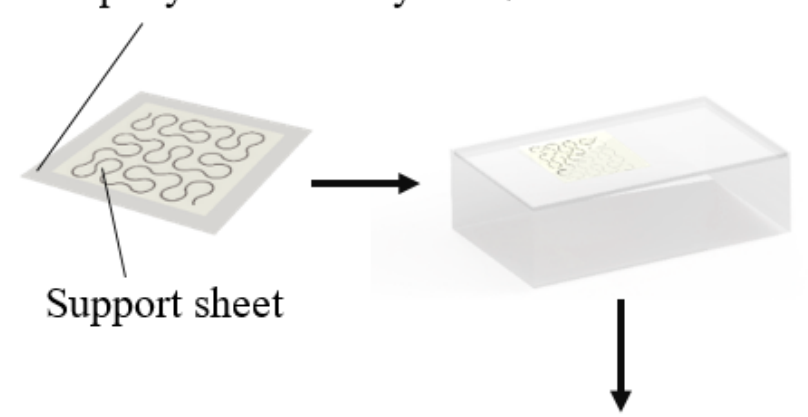

e)

d)

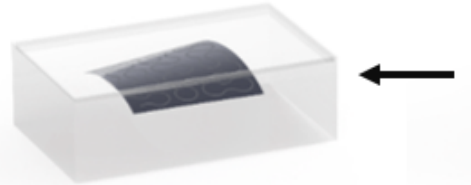

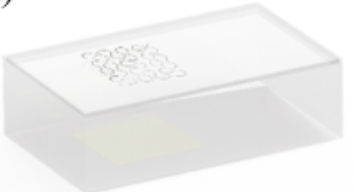

Figure 2. Fabrication processes of hydrographic printing. (a) materials are processed by laser pattern and screen-printing; (b) adhesive is attached to the screen-printing pattern; (c) complex is soaked in water; (d) paper layer of the support detaches from the complex; (e) left flexible film is transferred to a 3D object or skin; (f) finished electrode.

After finishing the electrode, scanning electronic microscopy (SEM) and energy dispersive X-ray spectroscopy (XPS) were taken to examine the surface and material characteristics of the traces. As shown in Figure $3 b$, the thickness of the silver-indium-gallium trace only adds by $17 \mu \mathrm{m}$ in contrast to only silver paste system, but the silver-indium-gallium trace has more pores after treated with the weak acid solution, as shown in Figure 3a. Hence, it's better to guarantee the cleaning process fulfilling in a very short time. The XPS survey spectra is shown in Figure 4a. The spectrum shows gallium, silver and indium peaks [44,45], which are zoomed in and shown in Figure $4 \mathrm{~b}-\mathrm{d}$, respectively. For $\mathrm{Ga}$, peaks are observed at $\sim 14.3 \mathrm{ev}$ and $20.1 \mathrm{ev}$, which associates with silver-indium-gallium system and oxidation. For Ag, peaks are observed at $362.8 \mathrm{ev}$ and $368.4 \mathrm{ev}$, which associates with silver-indium-gallium system and oxidation. For In, peak is observed at $\sim 451.8 \mathrm{ev}$, which associates with silver indium-gallium system. These results indicate that the system will lead to oxidation that can lower down the conductivity. Weak acid solution usually is applied to eliminate the influence [38]. 


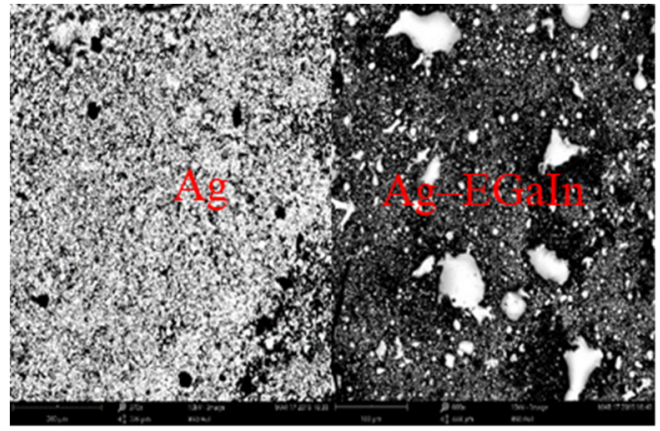

(a)

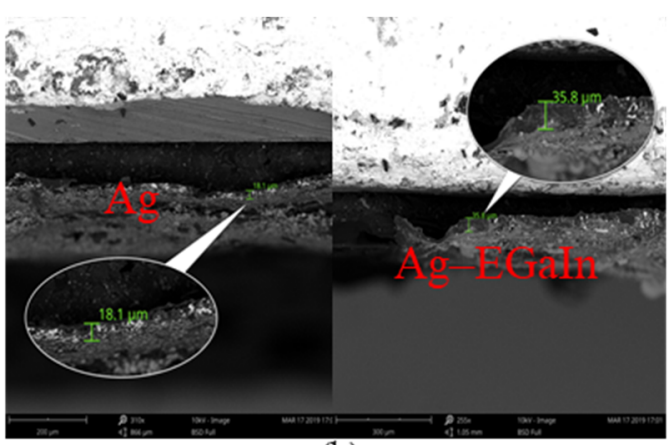

(b)

Figure 3. Characteristics of scanning electronic microscopy (SEM) (a) left is the only silver trace surface and the right is the Ag-EGaIn trace surface (b) right is the silver trace cross section and the left is the Ag-EGaIn trace cross section.

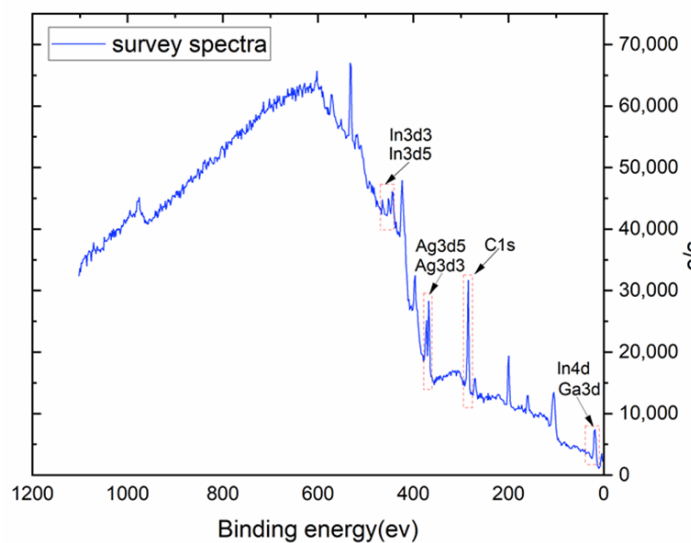

(a)

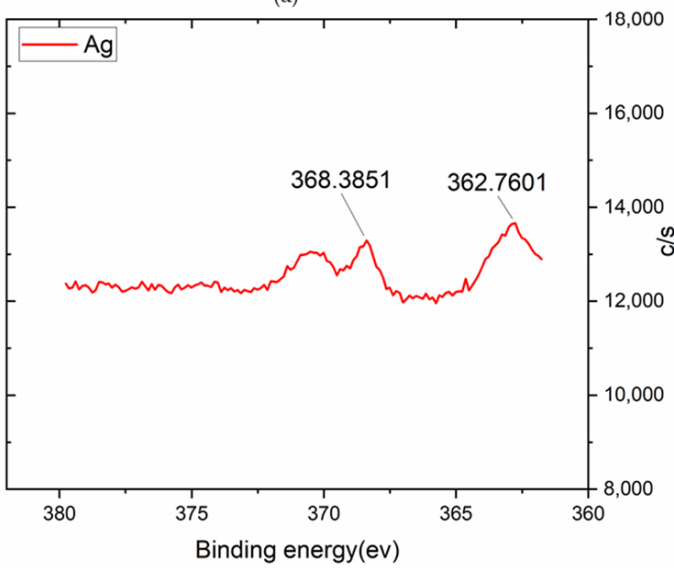

(c)

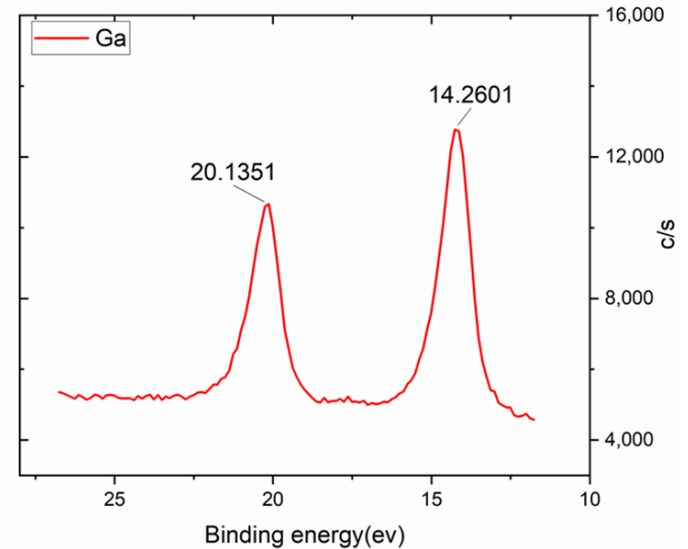

(b)

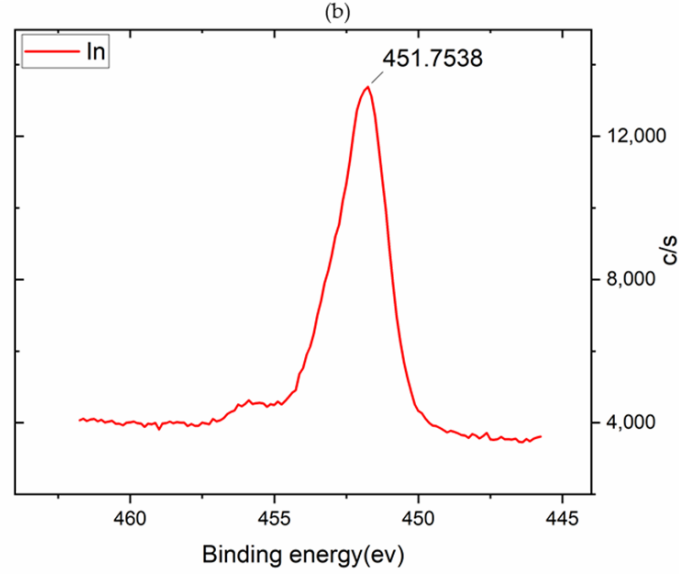

(d)

Figure 4. Characteristics of energy dispersive X-ray spectroscopy (XPS) (a) survey spectra; (b) binding energy of gallium; (c) the binding energy of silver (d) the binding energy of indium.

\section{Test results and Discussion}

\subsection{Electrical and Mechanical Performances}

The ability of electrodes to record sEMG depends strongly on the skin-electrode contact impedance. Comparative experiments between traditional wet electrode and the flexible dry electrode are carried out, which demonstrates that the contact impedance declines with the increasing intimacy. Figure 5 shows the practical application scenarios, the skin contact models and the equivalent circuits of the electrodes. The relative impedance data can be analyzed with the equivalent circuit that consists of electrode's resistor and coupling circuit. As illustrated in Figure 6, the tested contact impedance 
between skin and the flexible dry electrode is lower than that of the traditional wet electrode under $1000 \mathrm{~Hz}$ in which sEMG signal mainly distributes. Moreover, the impedance of the flexible electrode is much lower than that of the traditional electrode under $500 \mathrm{~Hz}$, which is the central power range of sEMG. The result proves that the flexible electrode is mounted much more intimately on skin.

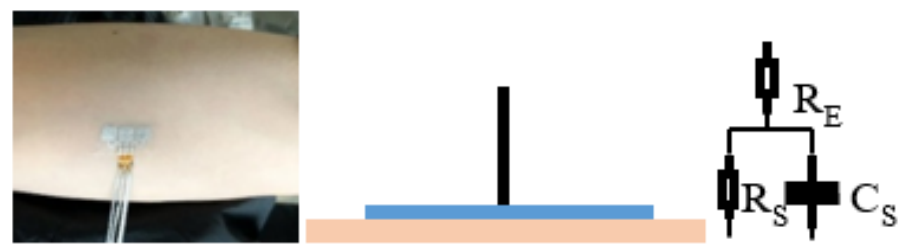

(a)

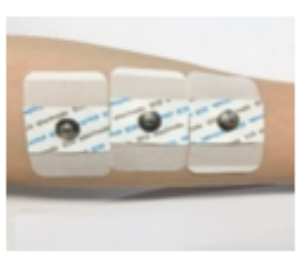

I

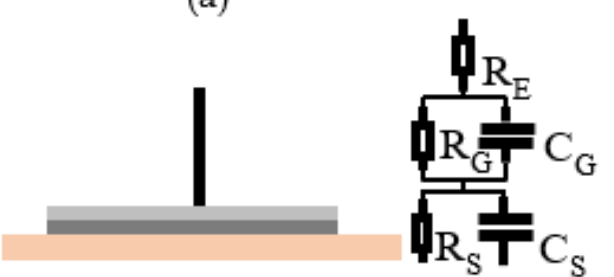

(b)

\section{Flexible electrode Skin Conductive gel Traditional electrode}

(c)

Figure 5. Comparative experiments between the traditional electrode and the flexible electrode. $\left(\mathrm{R}_{\mathrm{E}}\right.$ : resistance of electrode, $\mathrm{R}_{\mathrm{G}}$ : resistance of gel, $\mathrm{C}_{\mathrm{G}}$ : couple of gel, $\mathrm{R}_{\mathrm{S}}$ : resistance of skin, $\mathrm{C}_{\mathrm{S}}$ : couple of skin); (a) practical application scenario (left), the skin contact model (middle) and the equivalent circuit (right) of the flexible electrodes; (b) practical application scenario (left), the skin contact model (middle) and the equivalent circuit (right) of the traditional electrode; (c) illustration of the above.

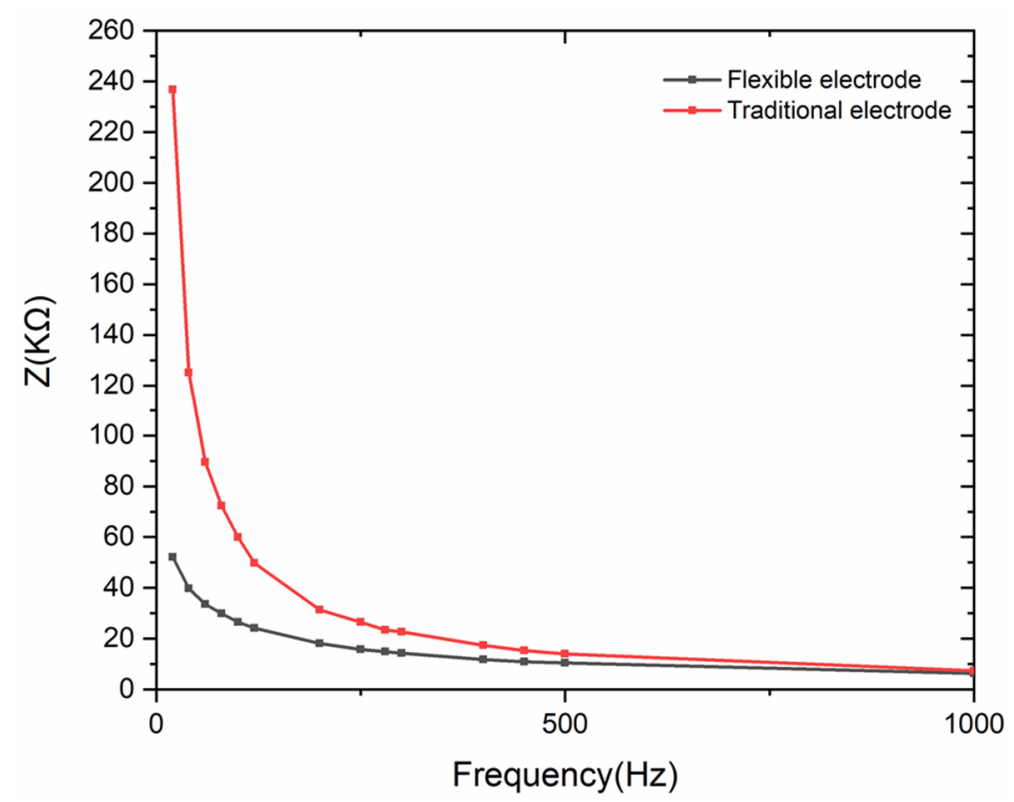

Figure 6. Tested contact impedance between skin and the electrodes at frequency range of 0-1000 Hz.

To better understand the influence of the mechanical deformation on electrical characteristics, the resistance of an eight-millimeter trace under stretch was examined. Referring to Figure $7 \mathrm{a}-\mathrm{c}$, the measured resistance shows a different performance in the different strain ratio. The lower strain ratio presents the better repeatability. However, the resistance always keeps at extremely low level. For each loading cycle, the peak resistance increases with the increased displacement, but produced regression 
and sharp dropping under $30 \%$ strain ratio experiments. These unexpected circumstances are mainly due to the postponement of the measurement, inertia existing in the trace under relaxing and irreversible deformation under great strain ratio. Overall, according to the relative resistance change (Figure 7d) as a function of strain, the trace still maintains excellent electrical characteristics without enormous variation even though the exact resistance has a slight rising. In contrast, this flexible electrode enables intimately and conformably mounting to skin with good electromechanical characteristics.
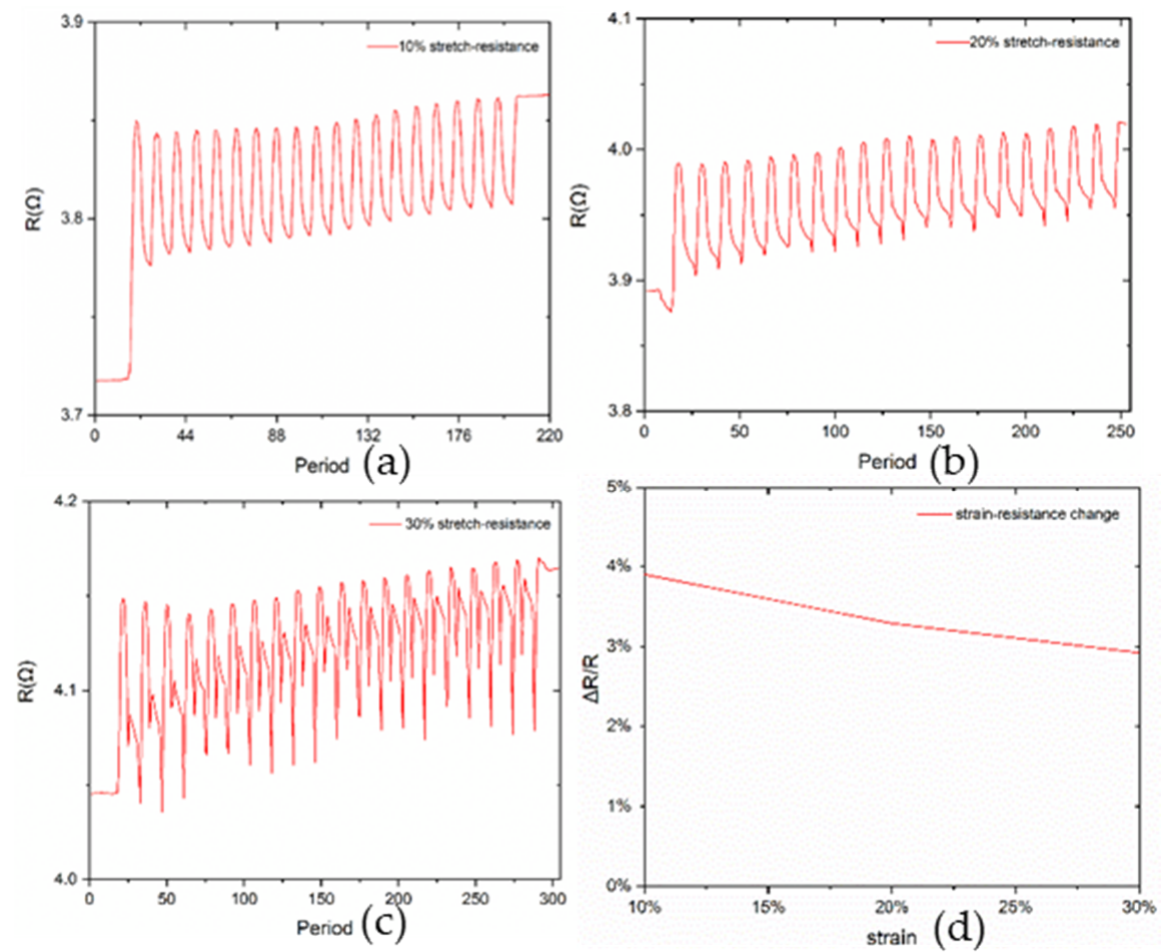

Figure 7. Stretch-resistance test under 10\%, 20\% and 30\% strain for 10 cycles and the relationship between strain and resistance change. (a) stretch-resistance test under 10\% strain; (b) stretch-resistance test under $20 \%$ strain; (c) stretch-resistance test under 30\% strain; (d) strain-resistance change relationship of the system.

\section{2. sEMG Monitoring Tests}

The electrical and mechanical features are summarized above as the fundamental for the sEMG signal measuring. The primary application test is to measure sEMG signal of biceps under voluntary contraction [46]. Three contrast experiments were taken to justify the practical signal quality of the flexible electrode. The Biopac MP36 sEMG scope was used to record the sEMG signal and two channels were separately connected to the traditional wet electrode and the flexible dry electrode at the same time. First, the electrodes measure 5 fist moves with the proper interval and the results are shown in Figure 8a and Figure S1. Second, the movements are continuous fists without intervals and the results were shown in Figure 8b and Figure S2. Third, the movements include not only the fist, but also the arm flex and the results were shown in Figure 8c and Figure S3. It shows that the flexible electrodes captured the signals of the muscle fiber precisely. However, the noise of the baseline is higher than the commercial traditional electrodes due to the unshielded connector between the flexible electrode leading wire and the Biopac MP36 sEMG scope. Nevertheless, the flexible electrodes are enough suitable to sEMG signal recording. 


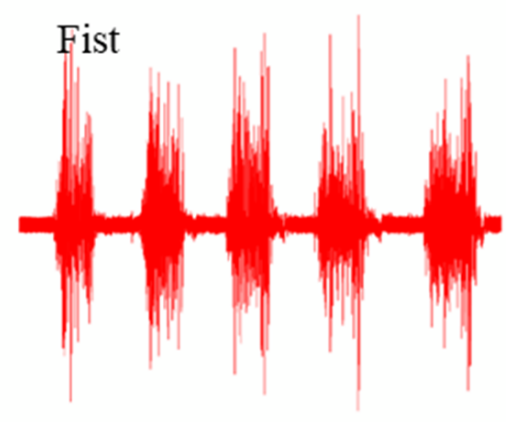

Traditional electrode

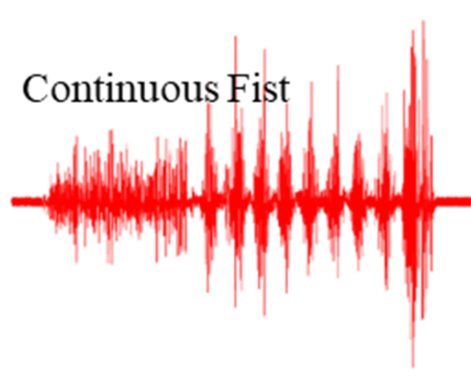

Traditional electrode

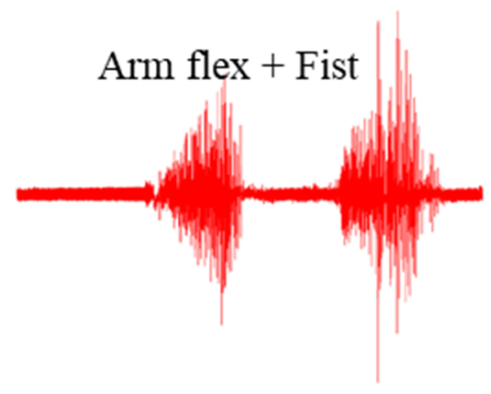

Traditional electrode

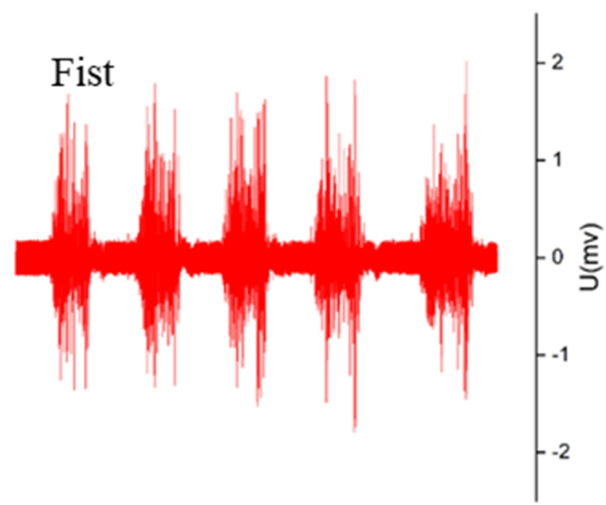

Flexible electrode

(a)

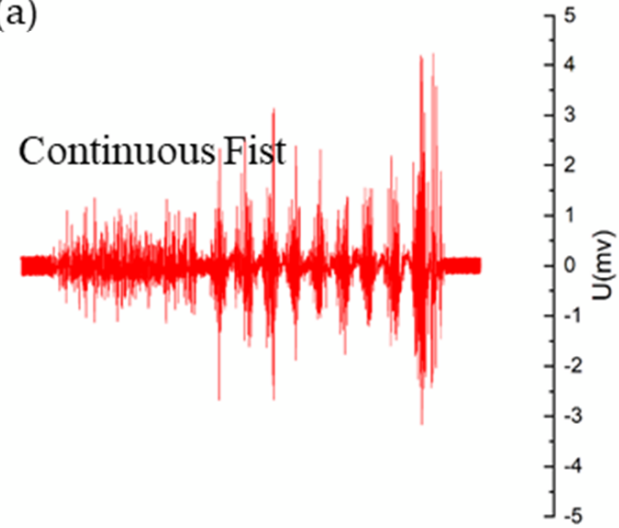

(b)

Flexible electrode

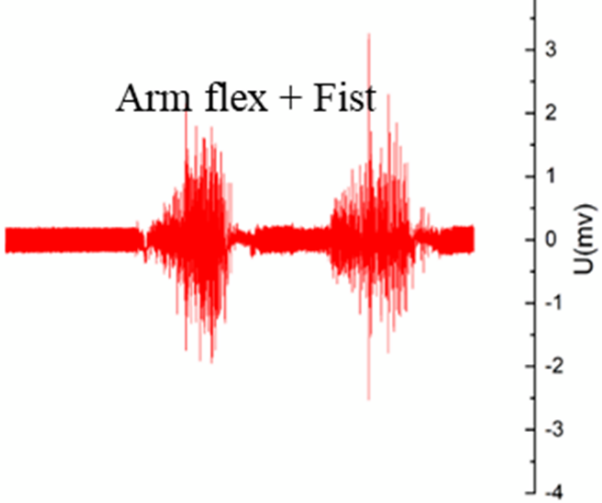

(c)

Figure 8. Comparative experiment of sEMG measurements by the traditional wet electrode (left) and the flexible dry electrode (right) (a) 5 fist moves with 2-s interval; (b) continuous fist moves without interval (c) arm flex and first at the same time.

\subsection{Wearability}

In addition to mechanical and electrical property tests, the conformable long-term wearability also takes a critical role in sEMG monitoring. Figure 9 shows the comparison of the wearable effects between the flexible and traditional electrodes. The skin zones of the traditional electrodes appear redness and swelling after the sEMG monitoring experiments. However, the more comfortable flexible electrodes only leave the shadow of the excess liquid metal. 


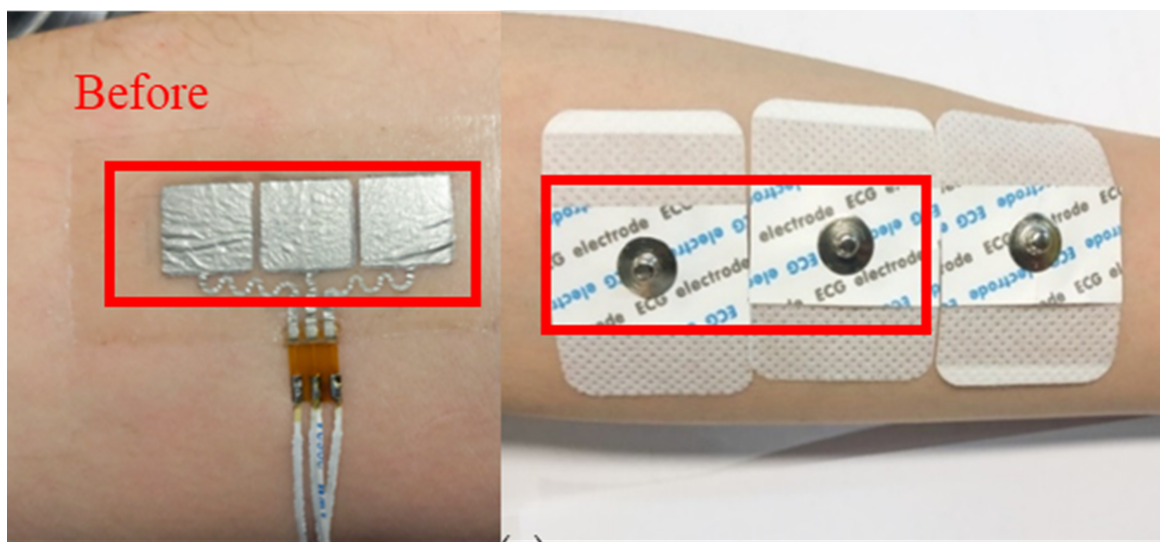

(a)

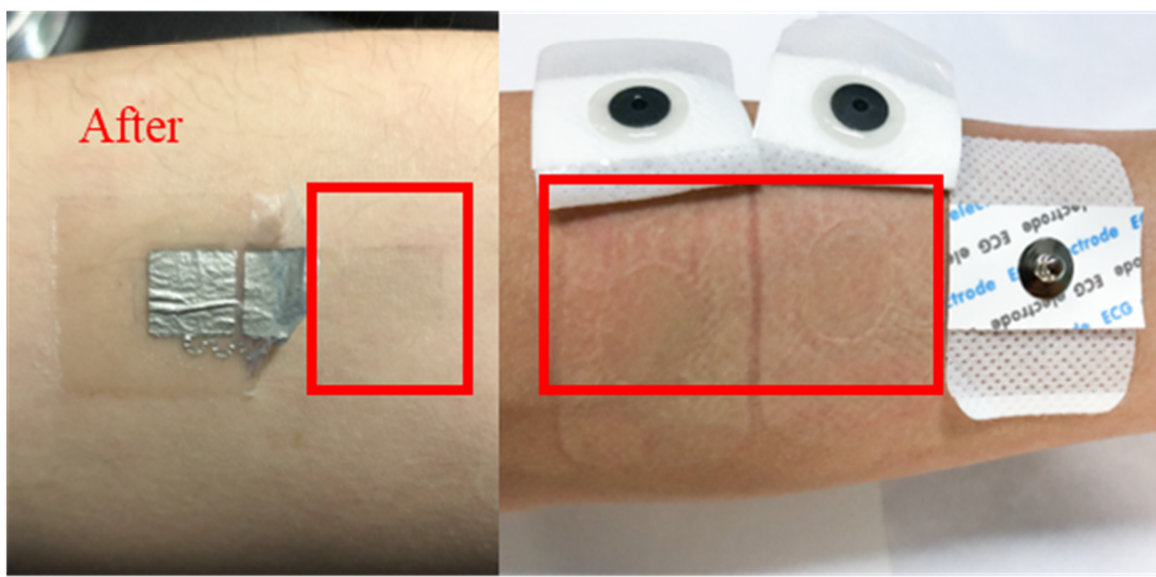

(b)

Figure 9. Skin reaction of traditional (right) and flexible (left) electrodes after sEMG measurements (a) presentation of the flexible electrodes and the traditional electrodes before the experiments; (b) presentation of the skin contact areas after the experiments.

\section{Conclusions}

An ultrathin, flexible and skin conformable dry electrode that can be applied without skin preparation was presented. The electrode is fabricated through hydrographic printing which can magnificently lower the cost and simplify the processing. Due to the novel structure and elaborate pattern, the electrode can susceptibly attach to the skin like a tattoo. This unique feature assures better signal quality and long-term wearability of the electrode in sEMG recording. In the comparative tests, the flexible dry electrode performs equally well or even better than traditional wet electrode, especially on aspects of skin-electrode contact impedance and wearability. Even though the ACF connector used in the experiments introduces a baseline noise, the dry electrode performs well under movement. In conclusion, the flexible dry electrode can be expected for sEMG monitoring applications.

Supplementary Materials: The following are available online at http://www.mdpi.com/1996-1944/13/10/2339/s1, Figure S1: movement of fist (a) the sEMG of fist 1; (b) the sEMG of fist 2; (c) the sEMG of fist 3, Figure S2: movement of continuous fist (a) the sEMG of continuous fist 1; (b) the sEMG of continuous fist 2; (c) the sEMG of continuous fist 3, Figure S3: movements of arm flex combining fist (a) the sEMG of arm flex combining with fist 1; (b) the sEMG of arm flex combining with fist 2.

Author Contributions: Conceptualization, X.Z., Y.D. and X.W.; formal analysis, X.Z.; investigation, X.Z., Y.D.; resources, X.W.; data curation, X.Z.; writing-original draft preparation, X.Z.; writing-review and editing, Y.D.; supervision, Y.D. and X.W.; funding acquisition, Y.D. All authors have read and agreed to the published version of the manuscript. 
Funding: This work is funded by the National Natural Science Foundation of China (Grant No. 61971262) and Natural Science Foundation of Guangdong Province (2020A1515010618).

Acknowledgments: Device fabrication and development were carried out in part in the NFC Tsinghua Shenzhen International Graduate School. The materials and device engineering aspects of the research were supported by the center of material and devices at Tsinghua Shenzhen International Graduate School. The authors would like to thank Yixin Liu for valuable discussion on manufacture processes.

Conflicts of Interest: The authors declare no conflict of interest.

\section{References}

1. Hong, Y.J.; Jeong, H.; Cho, K.W. Wearable and Implantable Devices for Cardiovascular Healthcare Monitoring to Therapy Based on Flexible and Stretchable Electronics. Adv. Funct. Mater. 2019, 29, 1808247. [CrossRef]

2. Liu, Y.; Pharr, M.; Salvatore, G.A. Lab-on-Skin: A Review of Flexible and Stretchable Electronics for Wearable Health Monitoring. ACS Nano 2017, 11, 9614-9635. [CrossRef]

3. Kim, D.H.; Lu, N.; Ma, R. Epidermal electronics. Science 2011, 333, 838-843. [CrossRef] [PubMed]

4. Huang, S.; Liu, Y.; Zhao, Y. Flexible Electronics: Stretchable Electrodes and Their Future. Adv. Funct. Mater. 2018, 29, 1805924. [CrossRef]

5. Zhang, D.; Sun, B.; Huang, H.; Gan, Y.; Xia, Y.; Liang, C.; Zhang, W.; Zhang, J. A Solar-Driven Flexible Electrochromic Supercapacitor. Materials 2020, 13, 1206. [CrossRef]

6. Shustak, S.; Inzelberg, L.; Steinberg, S. Home monitoring of sleep with a temporary-tattoo EEG, EOG and EMG electrode array: A feasibility study. J. Neural Eng. 2019, 16, 026024. [CrossRef]

7. Stauffer, F.; Thielen, M.; Sauter, C. Skin Conformal Polymer Electrodes for Clinical ECG and EEG Recordings. Adv. Healthc. Mater. 2018, 7, e1700994. [CrossRef] [PubMed]

8. Yeo, W.H.; Kim, Y.S.; Lee, J. Multifunctional epidermal electronics printed directly onto the skin. Adv. Mater. 2013, 25, 2773-2778. [CrossRef]

9. Wang, L.-F.; Yang, C.-S.; Yang, B. MEMS-based flexible capacitive electrode for ECG measurement. Electron. Lett. 2013, 49, 739-740. [CrossRef]

10. Pozzo, M. Electromyography (EMG), Electrodes and Equipment for. Wiley Encycl. Biomed. Eng. 2006. [CrossRef]

11. Wu, Y.; Martnez Martnez, M.; Orizaola Balaguer, P. Overview of the Application of EMG Recording in the Diagnosis and Approach of Neurological Disorders. In Electrodiagnosis in New Frontiers of Clinical Research, 1st ed.; IntechOpen: London, UK, 2013; Volume 10, p. 5772.

12. Mills, K.R. The basics of electromyography. J. Neurol. Neurosurg. Psychiatry 2005, 76 (Suppl. 2), 32-35. [CrossRef] [PubMed]

13. Kim, J.-E.; Seok, J.M.; Ahn, S.-W. Basic concepts of needle electromyography. Ann. Clin. Neurophysiol. 2019, 21, 7-15. [CrossRef]

14. Merletti, R.; Farina, D.; Holobar, A. Surface Electromyography (sEMG). Wiley Encycl. Electr. Electron. Eng. 2018, 1-22. [CrossRef]

15. Tian, L.; Zimmerman, B.; Akhtar, A. Large-area MRI-compatible epidermal electronic interfaces for prosthetic control and cognitive monitoring. Nat. Biomed. Eng. 2019, 3, 194-205. [CrossRef] [PubMed]

16. Jeong, J.W.; Yeo, W.H.; Akhtar, A. Materials and optimized designs for human-machine interfaces via epidermal electronics. Adv. Mater. 2013, 25, 6839-6846. [CrossRef] [PubMed]

17. Inzelberg, L.; Pur, M.D.; Schlisske, S. Printed facial skin electrodes as sensors of emotional affect. Flex. Print. Electron. 2018, 3, 045001. [CrossRef]

18. Srinivasa, M.G.; Pandian, P.S. Dry electrodes for bio-potential measurement in wearable systems. In Proceedings of the 2nd IEEE International Conference on Recent Trends in Electronics, Information \& Communication Technology, Sri Venkateshwara Coll Engn, Bangalore, India, 19-10 May 2017. [CrossRef]

19. Rockwood, D.N.; Preda, R.C.; Yucel, T. Materials fabrication from Bombyx mori silk fibroin. Nat. Protoc. 2011, 6, 1612-1631. [CrossRef]

20. Jung, H.C.; Moon, J.H.; Baek, D.H. CNT/PDMS composite flexible dry electrodes for long-term ECG monitoring. IEEE Trans Biomed. Eng. 2012, 59, 1472-1479. [CrossRef] 
21. Xu, B.; Akhtar, A.; Liu, Y. An Epidermal Stimulation and Sensing Platform for Sensorimotor Prosthetic Control, Management of Lower Back Exertion, and Electrical Muscle Activation. Adv. Mater. 2016, 28, 4462-4471. [CrossRef]

22. Kim, N.; Lim, T.; Song, K. Stretchable Multichannel Electromyography Sensor Array Covering Large Area for Controlling Home Electronics with Distinguishable Signals from Multiple Muscles. ACS Appl. Mater. Interfaces 2016, 8, 21070-21076. [CrossRef]

23. Yao, S.; Zhu, Y. Wearable multifunctional sensors using printed stretchable conductors made of silver nanowires. Nanoscale 2014, 6, 2345-2352. [CrossRef] [PubMed]

24. Ryu, S.; Lee, P.; Chou, J.B. Extremely Elastic Wearable Carbon Nanotube Fiber Strain Sensor for Monitoring of Human Motion. ACS Nano 2015, 9, 5929-5936. [CrossRef] [PubMed]

25. Abu-Saude, M.; Consul-Pacareu, S.; Morshed, B.I. Feasibility of patterned vertical CNT for dry electrode sensing of physiological parameters. In Proceedings of the 2015 IEEE Topical Conference on Biomedical Wireless Technologies, Networks, and Sensing Systems (BioWireleSS), San diego, CA, USA, 25-28 January 2015. [CrossRef]

26. Zhang, H.; Yang, S.; Sheng, K. The Leakage Mechanism of the Package of the AlGaN/GaN Liquid Sensor. Materials 2020, 13, 1903. [CrossRef] [PubMed]

27. Xia, N.; Li, N.; Rao, W. Multifunctional and Flexible ZrO2-Coated EGaIn Nanoparticles for Photothermal Therapy. Nanoscale 2019, 11, 10183-10189. [CrossRef]

28. Ozutemiz, K.B.; Wissman, J.; Ozdoganlar, O.B. EGaIn-Metal Interfacing for Liquid Metal Circuitry and Microelectronics Integration. Adv. Mater. Interfaces 2018, 5, 1701596. [CrossRef]

29. Green Marques, D.; Alhais Lopes, P. Reliable interfaces for EGaIn multi-layer stretchable circuits and microelectronics. Lab Chip 2019, 19, 897-906. [CrossRef]

30. Norton, J.J.; Lee, D.S.; Lee, J.W. Soft, curved electrode systems capable of integration on the auricle as a persistent brain-computer interface. Proc. Natl. Acad. Sci. USA 2015, 112, 3920-3925. [CrossRef]

31. Jang, K.I.; Han, S.Y.; Xu, S. Rugged and breathable forms of stretchable electronics with adherent composite substrates for transcutaneous monitoring. Nat. Commun. 2014, 5, 4779. [CrossRef]

32. Sirvent, V.F. Space filling curves and geodesic laminations. Geom. Dedicata 2008, 135, 1-14. [CrossRef]

33. Nawrocki, R.A.; Jin, H.; Lee, S. Self-Adhesive and Ultra-Conformable, Sub-300 nm Dry Thin-Film Electrodes for Surface Monitoring of Biopotentials. Adv. Funct. Mater. 2018, 28, 1803279. [CrossRef]

34. Khan, Y.; Pavinatto, F.J.; Lin, M.C. Inkjet-Printed Flexible Gold Electrode Arrays for Bioelectronic Interfaces. Adv. Funct. Mater. 2016, 26, 1004-1013. [CrossRef]

35. Jinno, H.; Fukuda, K.; Xu, X. Stretchable and waterproof elastomer-coated organic photovoltaics for washable electronic textile applications. Nat. Energy 2017, 2, 780-785. [CrossRef]

36. Hong, S.; Lee, S.; Kim, D.-H. Materials and Design Strategies of Stretchable Electrodes for Electronic Skin and Its Applications. Proc. IEEE 2019, 107, 1-13. [CrossRef]

37. Saada, G.; Layani, M.; Chernevousky, A. Hydroprinting Conductive Patterns onto 3D Structures. Adv. Mater. Technol. 2017, 2, 1600289. [CrossRef]

38. Lopes, P.A.; Paisana, H.; De Almeida, A.T. Hydroprinted Electronics: Ultrathin Stretchable Ag-In-Ga E-Skin for Bioelectronics and Human-Machine Interaction. ACS Appl. Mater. Interfaces 2018, 10, 38760-38768. [CrossRef]

39. Linghu, C.; Zhang, S.; Wang, C. Transfer printing techniques for flexible and stretchable inorganic electronics. Flex. Electron. 2018, 2, 1-14. [CrossRef]

40. Tavakoli, M.; Malakooti, M.H.; Paisana, H. EGaIn-Assisted Room-Temperature Sintering of Silver Nanoparticles for Stretchable, Inkjet-Printed, Thin-Film Electronics. Adv. Mater. 2018, 30, 1801852. [CrossRef]

41. Wang, Q.; Ling, S.; Liang, X. Self-Healable Multifunctional Electronic Tattoos Based on Silk and Graphene. Adv. Funct. Mater. 2019, 29, 1808695. [CrossRef]

42. Fan, J.A.; Yeo, W.H.; Su, Y. Fractal design concepts for stretchable electronics. Nat. Commun. 2014, 5, 3266. [CrossRef]

43. Campbell, A.N.; Reynolds, W.F. THE SYSTEM SILVER-INDIUM-GALLIUM. Can. J. Chem. 1962, 40, 37-45. [CrossRef]

44. Liu, S.; Yuen, M.C.; White, E.L. Laser Sintering of Liquid Metal Nanoparticles for Scalable Manufacturing of Soft and Flexible Electronics. ACS Appl. Mater. Interfaces 2018, 10, 28232-28241. [CrossRef] [PubMed] 
45. Cademartiri, L.; Thuo, M.M.; Nijhuis, C.A. Electrical Resistance of AgTS-S(CH2)n-1CH3//Ga2O3/EGaIn Tunneling Junctions. J. Phys. Chem. C 2012, 116, 10848-10860. [CrossRef]

46. Raez, M.B.; Hussain, M.S.; Mohd-Yasin, F. Techniques of EMG signal analysis: Detection, processing, classification and applications. Biol. Proced. Online 2006, 8, 11-35. [CrossRef] [PubMed]

(C) 2020 by the authors. Licensee MDPI, Basel, Switzerland. This article is an open access article distributed under the terms and conditions of the Creative Commons Attribution (CC BY) license (http://creativecommons.org/licenses/by/4.0/). 